\title{
FOUNDATION OF VORTEX GRAVITATION, COSMOLOGY AND COSMOGONY
}

\author{
S. A. Orlov \\ Petrozavodsk State University \\ Corresponding author: ion@ onego.ru \\ Received 1 April, 2010; Revised 19 October, 2010
}

A hypothesis is proposed concerning the cause of the origin of universal gravitation. This cause consists in a system of the ether vortex rotations. Physical and mathematical grounds are described and the formula for the determination of the space gravitation forces is deduced. On the basis of the vortex gravitation, the principles of creation and existence of the celestial bodies are shown. Methods of the use of the vortex gravitation properties for the space flight projection are proposed.

Keywords: $\quad$ Ether Theory, Vortex Gravitation, Cosmology and Cosmogony

\section{INTRODUCTION}

The proposed model of gravitation, cosmology and cosmogony is based on an assumption that the initial cause of rotations of all the celestial objects or their systems in the Universe is the vortex rotation (in corresponding space regions) of a gaseous cosmic-space medium, viz. the ether.The ether rotation occurs according to the same law as the rotation of a celestial body. In the solar system (torsion), the vortex-type ether rotation has the torsion character and corresponds to the circulation of the planets around the Sun (the Kepler 3-rd law). The torsion-like vortex ether rotation creates an ether pressure change inside the torsion in accordance with the aerodynamics laws. The pressure gradient in the space medium creates the expulsive force acting onto the bodies located in this medium, and this force is directed toward the center of the torsion.

\section{The expulsive force is just the gravity force.}

Calculations of the expulsive (gravity) forces is carried out on the basis of mechanics of continua and (or) aerodynamics with the use of the Navier-Stokes equations. In the present work, an algebraic formula for the gravitation forces is obtained, which does not contain reduce coefficients, likewise the gravitation constant in the Newton formula. In the article, some conclusions and foundations are proposed, as well as novel methods for investigation of the numerous properties of the celestial bodies of the cosmic substance, such as:

1. Universal vortex gravitation has a discoid configuration, which is confirmed by some astronomy facts, e.g., by ellipsoidal planet orbits. If one takes into account this property of gravitation, this allows an explanation of the inaccuracy of the commonly-accepted Universe Gravity (Newtonian attraction) Equation. Also, this allows obtaining of a correction for the twodimensional Newton's formula. On the basis of the obtained three-dimensional formula for the determination of the gravitation forces, a possibility appears to plan cosmic flights with a significant decrease of the energy consumption.

2. Based on the principle of vortex gravitation, physical models are developed in the article, in which different properties of the cosmic objects, as well as the principles of creation of the universal substance and celestial objects, Black Holes, the character of their motion, masses, age, evolution of stars, planets, and the Universe in the whole, are presented. 
3. Foundations of the distance increase between the galaxies are proposed, and these foundations are in fact the confirmations of the Universe contraction. With the help of the physical model of vortex gravitation, the causes of ebbs and flows in the oceans are explained.

4. On the basis of the model of vortex gravitation, analytical explanations of the numerous scientific paradoxes has been suggested:

- Paradox of Seliger, the Earth rotation velocity decrease, existence of "dark matter", etc. Advantages of the proposed model of gravitation as compared with the Newtonian formula consist in the fact that it determines the gravity forces using the commonly-recognized basic physical properties and laws. Meanwhile, the Newton's law is based only on the experimental, observable facts. Therefore, on the basis of the vortex gravitation model, a possibility appears to account for the nature of the gravitation and to work out technological methods for an affect onto the gravity forces.

A huge number of thinkers pointed out the main role of the cosmic vortexes in the process of the world substance creation. In the Ancient World they were Empedocles, Leucippus, Democritus, Aristotle and some others. In the Renascence this idea was developed by R. Descartes, J. MacCullagh, J. J. Thomson, and W. Thomson (Lord Kelvin).It should be noted that that times there was no a developed science concerning continua. Mathematics and physics were not enriched yet with the works by Bernoulli, Euler, Navier, Stoke, etc. Therefore, the above mentioned scientists could not formulate their ideas in a physical and mathematical form and their findings did not go out from the purely philosophy speculations. In $20^{\text {th }}$ century, the substance "ether" was groundlessly removed from the scientific consideration as if it was being «pseudo-scientific», which stopped the development of the theory of the space ether vortexes for long years. In the present article, we present numerous evidences of the existence of vortex gravitation, sufficient to consider it as a working hypothesis or a theory. The evidences are proposed for consideration all over the text below.

\subsection{MODEL OF THE ORIGIN OF THE UNIVERSAL GRAVITATION FORCE}

In this section, a model of appearance of the gravitation attraction force is considered from the viewpoint of aerodynamics. Namely, the two-dimensional model (Fig.1) is considered on the basis of the following initial postulates. These postulates will be expanded and defined more exactly below.

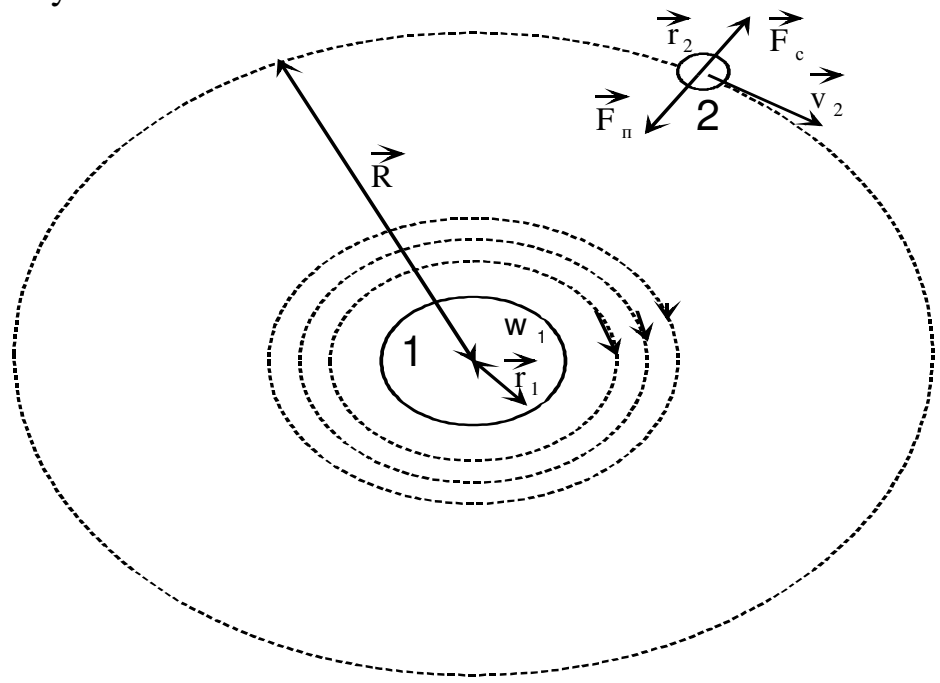

Fig.1. Two-dimensional model of gravitational interaction of two bodies. The forces are shown acting on body $2: F_{c}-$ the centrifugal force, $F_{\Pi}$ - the force of attraction of body 2 from body $1 ; v_{2}$ - linear velocity of body 2 at the orbit, $\mathrm{R}$ - the radius of the orbit, $\mathrm{r}_{1}$ - the radius of body $1, \mathrm{r}_{2}-$ the radius of body $2, \mathrm{w}_{1}$ - angular velocity of ether rotation at the surface of body 1 . 
1. There exists an ether vortex around any physical object.

2. The ether motion in the vortex has laminar nature and obeys the laws of hydro- or aerodynamics; the ether viscosity is low.

3 . The pressure gradient, arising during the vortex motion of the ether gas, is the reason for an attractive force from body 1 to body 2 (see Fig.1).

4. The direction of the force $\mathbf{F}_{\mathbf{n}}$ does not depend on the direction of the ether angular velocity, which is necessary for the attractive force between the bodies, irrespective of their relative position. This implies the absence of the Magnus force - the force of interaction between the two vortexes which appears in the classical aerodynamics. Such an assumption can take place at a weak interaction between the two ether flows, as if they would move one through another, not affecting mutual motion.

5. The appearing attraction force must describe the experimentally obtained law of gravity:

$$
\mathrm{F}_{\Pi}=\mathrm{G} \cdot \frac{\mathrm{m}_{1} \cdot \mathrm{m}_{2}}{\mathrm{r}^{2}}
$$

where $\mathrm{m}_{1}, \mathrm{~m}_{2}$ are the masses of bodies 1 and 2 , respectively, $\mathrm{G}=6.672 \cdot 10^{-11} \mathrm{~N} \cdot \mathrm{m}^{2} / \mathrm{kg}^{2}-$ the gravitation constant, and $\mathrm{r}-$ the distance between the bodies.

Next we consider the appearance of the attraction force in more detail and derive a formula describing it. As was said above, a pressure gradient arises as the result of the vortex motion. Let's find the radial distribution of the pressure and the ether velocity. For this purpose, we write the Navier-Stokes equation for the motion of a viscous liquid (gas).

$$
\rho\left[\frac{\partial}{\partial \mathrm{t}}+\overrightarrow{\mathrm{V}} \cdot \operatorname{grad}\right] \overrightarrow{\mathrm{v}}=\overrightarrow{\mathrm{F}}-\operatorname{grad} \mathrm{P}+\eta \Delta \overrightarrow{\mathrm{v}}
$$

where $\rho$ is the ether density, $\vec{V}$ and $P$ are, respectively, its velocity and pressure, and $\eta-$ the ether viscosity. In cylindrical coordinates, taking into account the radial symmetry $\mathrm{v}_{\mathrm{r}}=\mathrm{v}_{\mathrm{z}}=0$, $\mathrm{v}_{\varphi}=\mathrm{v}(\mathrm{r}), \mathrm{P}=\mathrm{P}(\mathrm{r})$, the equation can be written as the system:

$$
\left\{\begin{array}{l}
-\frac{\mathrm{v}(\mathrm{r})^{2}}{\mathrm{r}}=-\frac{1}{\rho} \frac{d \mathrm{P}}{d \mathrm{r}} \\
\eta \cdot\left(\frac{\partial^{2} \mathrm{v}(\mathrm{r})}{\partial \mathrm{r}^{2}}+\frac{\partial \mathrm{v}(\mathrm{r})}{\mathrm{r} \partial \mathrm{r}}-\frac{\mathrm{v}(\mathrm{r})}{\mathrm{r}^{2}}\right)=0
\end{array}\right.
$$

In case of a compressible substance (ether), there will be a function $\rho=f(P)$ (instead of $\rho$ ).

From the first equation of system (3), one can find $\mathrm{P}(\mathrm{r})$ provided that the dependence $\mathrm{v}(\mathrm{r})$ is known. The latter, in turn, should be found from the second equation of that same system (one of the solution of which is the function $\mathrm{v}(\mathrm{r}) \sim 1 / \mathrm{r})$. At zero viscosity, the system permits any dependence $v(r)[2]$.

The force affecting the body can be estimated from the formula

$$
\overrightarrow{\mathrm{F}}_{\Pi}=-\mathrm{V} \bullet \operatorname{grad} \mathrm{P}(\mathrm{r})
$$

where $\mathrm{V}$ is the volume of body 2.

In cylindrical coordinates the modulus of $\vec{F}_{\Pi}$ is 


$$
\mathrm{F}_{\Pi}=\mathrm{V} \cdot \frac{\partial \mathrm{P}}{\partial \mathrm{r}}
$$

Then, comparing equations ( 3 ) and (5), for the incompressible ether ( $\rho=$ const) we find that

$$
\mathrm{F}_{\mathrm{\Pi}}=\mathrm{V} \cdot \rho \cdot \frac{\mathrm{v}(\mathrm{r})^{2}}{\mathrm{r}}
$$

For the correspondence of the ether rotation to the planet motion law (according to Kepler 3rd law) in one cosmic (e.g., Solar) system, v(r) must obey the dependence $v(r) \sim \frac{1}{\sqrt{r}}$, and not the $\mathrm{V}(\mathrm{r}) \sim \frac{1}{\mathrm{r}}$.

Taking into account the edge condition $\mathrm{v}\left(\mathrm{r}_{1}\right)=\mathrm{w}_{1} \cdot \mathrm{r}_{1}$,

$$
\mathrm{v}(\mathrm{r})=\frac{\mathrm{w}_{1} \cdot r_{1}^{\frac{3}{2}}}{\sqrt{\mathrm{r}}}
$$

Thus

$$
\mathrm{F}_{\mathrm{\Pi}}=\mathrm{V} \cdot \rho \cdot \frac{w_{1}^{2} \cdot r_{1}^{3}}{\mathrm{r}^{2}}
$$

Here we make one more supposition (№ 6) - Ether penetrates through all the space, including the physical bodies. The volume $\mathrm{V}$ in formula (8) is an effective volume, i.e. the volume of elementary particles, which the body is composed of. All the bodies are composed of electrons, protons, and neutrons. The radius of an electron is much smaller than that of a proton and neutron. The radii of the latter are approximately equal to each other, $\mathrm{r}_{\mathrm{n}} \sim 1.2 \cdot 10^{-15} \mathrm{~m}$. The same is true as to the masses: $\mathrm{m}_{\mathrm{n}} \sim 1.67 \cdot 10^{-27} \mathrm{~kg}\left(\mathrm{r}_{\mathrm{n}}\right.$ and $\mathrm{m}_{\mathrm{n}}$ are the radius and the mass of a nucleon). Therefore, the volume in formula (8) is:

$$
\mathrm{V}=\frac{\mathrm{m}_{2}}{\mathrm{~m}_{\mathrm{n}}} \cdot \frac{4 \pi}{3} \cdot \mathrm{r}_{\mathrm{n}}^{3}
$$

Taking into account the formula (9), Eq.(8) can be rewritten as

$$
\mathrm{F}_{\mathrm{\Pi}}=\frac{4 \cdot \pi \cdot \mathrm{r}_{\mathrm{n}}^{3} \cdot \rho}{3 \cdot \mathrm{m}_{\mathrm{n}}} \cdot \frac{w_{1}{ }^{2} \cdot r_{1}^{3} \cdot \mathrm{m}_{2}}{\mathrm{r}^{2}}
$$

Supposing further (supposition № 7) that

$$
\mathrm{w}_{1}{ }^{2} \cdot \mathrm{r}_{1}{ }^{3}=\mathrm{A} \cdot \mathrm{m}_{1}
$$

where $\mathrm{A}$ is a constant, Eq.(10) takes the form 


$$
\mathrm{F}_{\Pi}=\frac{4 \cdot \pi \cdot \mathrm{r}_{\mathrm{n}}^{3} \cdot \rho}{3 \cdot \mathrm{m}_{\mathrm{n}}} \cdot \mathrm{A} \cdot \frac{\mathrm{m}_{1} \cdot \mathrm{m}_{2}}{\mathrm{r}^{2}}
$$

Comparing equations (12) and (1), one can find that $A=1.739 \cdot 10^{18} \mathrm{~m}^{3} / \mathrm{s}^{2} \cdot \mathrm{kg}$. The data on the parameters of free ether used for calculations were taken from Ref.[1].

The supposition № 7 is reasonable, since $\mathrm{w}_{1}$ and $\mathrm{r}_{1}$ are the parameters of body 1 . If we divide both the left- and right-hand sides of Eq.(11) by $\mathrm{r}_{1}{ }^{3}$, we will obtain that the square of the ether angular velocity on the surface is proportional to the body's density. Let's find, e.g., the angular ether velocity on the surface of the Sun:

$$
\mathrm{w}_{1}=\sqrt{\mathrm{A} \cdot \frac{\mathrm{m}_{1}}{\mathrm{r}_{1}^{3}}}
$$

The mass of the Sun is $\mathrm{m}_{1}=1.99 \cdot 10^{30} \mathrm{~kg}, \mathrm{r}_{1}=6.96 \cdot 10^{8} \mathrm{~m}$, and $\mathrm{w}_{1}=1.022 \cdot 10^{11} \mathrm{c}^{-1}$.

The ether linear velocity on the surface is $\mathrm{v}\left(\mathrm{r}_{1}\right)=\mathrm{w}_{1} \cdot \mathrm{r}_{1}=7.113 \cdot 10^{19} \mathrm{~m} / \mathrm{s}$. This velocity is lower than the average speed of amers in ether $\left(6.6 \cdot 10^{21} \mathrm{~m} / \mathrm{s}\right.$ [1] $)$ by two orders of magnitude. Thus, the obtained value of the ether wind linear velocity appears to be quite reasonable. For Earth, $\mathrm{m}_{1}=5.98 \cdot 10^{24} \mathrm{~kg}, \mathrm{r}_{1}=6.38 \cdot 10^{6} \mathrm{~m}$, and $\mathrm{w}_{1}=2.001 \cdot 10^{11} \mathrm{~s}^{-1}, \mathrm{v}\left(\mathrm{r}_{1}\right)=1.277 \cdot 10^{18} \mathrm{~m} / \mathrm{s}$.

On the basis of vortex gravitation, the value of $\mathrm{w}_{1}$ in any celestial torsion is determined from the condition of the equality of the centrifugal forces and the gravitation forces for a celestial body.

Taking into account the compressibility of ether, e.g. in the isothermal case ( $\mathrm{T}=\mathrm{const})$, i.e. when

$$
\rho=f(P)=\frac{P}{R \cdot T}
$$

where $\mathrm{R}$ is the specific gas constant $\mathrm{R}=\frac{\mathrm{R}_{0}}{\mu}=\frac{\mathrm{R}_{0}}{\mathrm{~m}_{0} \cdot \mathrm{Na}}=1.972 \cdot 10^{93} \mathrm{~J} \cdot \mathrm{kg}^{-1} \cdot \mathrm{K}^{-1}\left(\mathrm{R}_{0}=8.314\right.$ $\mathrm{J} \cdot \mathrm{mol}^{-1} \cdot \mathrm{K}^{-1}$ - the absolute gas constant, $\mu$ - the ether molecular weight, $\mathrm{m}_{0}=7 \cdot 10^{-117} \mathrm{~kg}-$ the mass of an amer [1], $\mathrm{Na}=6.022 \cdot 10^{23} \mathrm{~mol}^{-1}$ - the Avogadro number), after the first equation in system (3) to be solved, we have got a function of the pressure radial distribution. This function, using e.g. the values of $\mathrm{w}_{1}$ and $\mathrm{r}_{1}$ for the Sun, results in a very insignificant change of the density with radius enabling the ether to be considered as an incompressible substance, and thereby, enabling the above-presented formulas to be used.

Let's now find the dependence $\mathrm{P}(\mathrm{r})$ solving the first equation of system (3). Taking Eq.(7) into account, we'll find that

$$
\mathrm{P}(\mathrm{r})=\mathrm{P}_{0}+\rho \cdot \mathrm{w}_{1}^{2} \cdot \mathrm{r}_{1}^{3} \cdot\left[\frac{1}{\mathrm{r}_{1}}-\frac{1}{\mathrm{r}}\right]
$$

where $\mathrm{P}_{0}$ is the ether surface pressure. Using the boundary condition $\mathrm{P}(\infty)=\mathrm{P}_{b}$, we get $\mathrm{P}_{0}=\mathrm{P}_{b}-\rho \cdot \mathrm{W}_{1}^{2} \cdot \mathrm{r}_{1}^{2}$ with $\mathrm{P}_{\mathrm{b}}$ being the pressure of free ether (Fig. 2) 




$\mathrm{r} 1 \cdot 20$

Fig. 2 Radial distribution of the ether pressure for the Sun

From the obtained formula for vortex gravitation, it is obvious that, in the existing Newton's law of gravitation, instead of the reason of gravity (the gradient of pressure), the consequence of that (i.e. the mass) is used.

\subsection{SPATIAL MODEL OF GRAVITATION}

In order to solve the posed problem of calculations of the forces of vortex gravitation, it is necessary to determine the space ether torsion configuration. In view of the very small ether density, it is impossible to study the ether by means of direct methods. However, since (on the basis of the ether cosmogony - see Section 3 below) the ether creates celestial bodies and transfers to them the momentum of their movement, then it is possible to determine the character of the ether rotation and its configuration using the form of the celestial objects and the character of their movement.

Because the main vortex shape characteristics are its diameter and thickness, the main objective of the investigation of the torsion configuration is just to determine these two parameters. The size of the torsion diameter is quite obvious and is equal to the diameter of the corresponding cosmic (solar) system. The latter is determined by the most distant orbits of the satellites of this torsion. At the same time, the vortex-like ether rotations occur in the cosmic space as some discoid media of a negligible (very small) thickness.

This suggestion is based on commonly-known astronomy facts.

1. Because all the celestial objects have spherical or ellipsoidal shapes, for the accumulation of the cosmic matter as a sphere (under the action of the vortex gravitation), it is needed that an active axis thickness of the vortex ether flow be less than the diameter of the created body. The point is that, if the ether would rotate as a "rotor" with a significant axis thickness, then such a rotor would produce the gravitation with cylindrical configuration. In this case, the cosmic matter indrawn by this torsion would have the same cylindrical form. Since as such the cylindrical shapes of the celestial objects are not observed, then the conclusion is obvious, namely, the ether rotation has a form of a "thin" rotating disc.

When comparing the value of diameter of any celestial object with the diameter of the corresponding space system (solar, planetary), it is obvious that the diameter of an object is negligibly small as compared with the system diameter, and consequently a vortex "cuts up" the cosmic space by a layer with an also negligibly small thickness.

2. All the systems of celestial objects (galaxies, systems of planets and satellites) circulate around a center in a unique orbital plane with small inclination. 
Such trajectories of the celestial systems are possible only in the case if the ether (in corresponding space regions) also rotates in the same planes with a negligibly small axes thickness cutting the massive ether. According to the aerodynamics laws, the pressure decreases in these planes, and all the bodies or substances from the neighboring regions are pushed out toward the central plane of this rotation and (or) are kept back in it.

3. In chapter 3.3 and 3.4 proofs are presented about the fact that based on dynamics laws, circulation of planets around Sun on elliptical orbits is possible only at go-symmetric, and not central- symmetric (classical) character action of powers of sunny gravitation. Kepler recognized action of powers attraction in solar system on inverse-square law only in planes ecliptic.

As plane of ecliptic has small deviation from плоско-сти of sunny, gravitational torsion bar, that its suggestion molybdenum-put very close to offered vortical, go-symmetric gravitation The most pictorial visual rendition of the cosmic torsion shape one can see looking at a galaxy as an example. The spiral-like armlets of galaxies earnestly show their vortex nature. Also, it is known from astronomy that the diameter and thickness of a galaxy has a ratio 10:1. Since this dependence corresponds to point $\mathbf{2}$ of the present Section above, it is quite obvious that this configuration of celestial torsions is the most typical in cosmic space. That is, stellar (e.g., solar) and planetary (e.g., Earth) ether torsions have the same discoid form.

\section{SOME CONCLUSIONS}

The model of the universal vortex gravitation determines absolutely new principles of the origination and existence of the universe substance.

The below-suggested calculations and results do not pretend to be complete and exact. The main purpose of Section 3 is to present evidences of the existence of vortex nature of the gravitation forces with its discoid configuration, as well as to show applied possibilities of the model of vortex gravitation for the principally new study of numerous cosmology phenomena. Any specialist can independently work out ways and methods of calculations and investigations on the basis of vortex gravitation.

\subsection{BLACK HOLES}

In 1783 John Mitchell has presented his work where he sowed that a sufficiently massive and compact star should have such a high gravitation field that light could not get it out. Such objects are called Black Holes. On the basis of the proposed model of vortex gravitation and obtained formula (10), and also taking into account the assumption №6 of Section 2, one can determine the gravitation force in any point of space, including that inside celestial objects, particularly inside the Sun.

It has been established by means of calculations that vortex gravitation, corresponding by its strength to the gravitation force of a Black Hole $(\mathrm{BH})$, exists in the sun torsion in a distance of 3 kilometers from the center. This distance corresponds to schwarzschild radius, but thus it is not required condensation of material of Sun to volume of such radius. Besides, on the basis of that same calculation, all the celestial torsions, including the planetary ones, should theoretically have a gravitation force, corresponding to that of the BH. A super-high gravitation force, caused by a corresponding pressure, creates in the centers of all celestial objects (including planets) a physical basis for the existence of thermal-nuclear reactions in these central zones.Hence, $\mathrm{BH}$ is a central part of the cosmic ether torsion which, possessing gravitation, creates a new celestial object. That is, BH is not a collapse of a celestial object, but it is a newly-formed cosmic torsion which does not possess a physical body yet. 
An outside observer can fix a Black Hole only at the moment when the center of the cosmic torsion is not still hidden by the cosmic substance which the torsion have to suck in starting from the moment of birth. After the concentration of cosmic substance in the center of the torsion in a volume sufficient to hid over-gravitation zone, this celestial object turns into a conventional celestial object - a planet, star, etc.

The super-massive BH in the center of our galaxy, rotating with a huge speed, convincingly supports the proposed concept of the nature of black holes.

\subsection{EVOLUTION OF STARS}

In modern astrophysics, the star evolution is considered according to the following scheme: - at the initial stage, the appearance of dense clouds of gas and dust occurs, from which, under the action of the own gravitation, a compressing proto-star is formed. Then, an ordinary star is formed, and after that it turns into a red giant, and further - into a white dwarf. As the concluding phase of the star existence, modern cosmology considers the stage of $\mathrm{BH}$ which is accompanied by the collapse of the celestial object.

It should be noted that in this hypothesis, the reasons for the appearance of the gas-dust matter, as well as those for substance and stars from this diffusive medium, are not indicated. Besides, the proposed process of the celestial objects self-densification has very weak argumentation. The star evolution based on the principle of vortex gravitation is quite the reverse as compared to the classical ideas; however, it is in complete conformity with the basic physical laws. As was shown in Section 3.1, any torsion in the initial stage, in its central zone, has a form of $\mathrm{BH}$. After the substance accumulation exceeding the volume of $\mathrm{BH}$, the gravitation forces on the outer layers of the created star decrease inversely proportionally with square of the distance from the torsion center. In spite of this, these forces preserve their great magnitude which compels the star substance to compress up to the neutron state. Consequently, the next star stage after the BH is the neutron star which, in turn, is subdivided into several phases. The initial phase of the neutron star is the pulsar. At present, the pulsar PSRJ1748-2446 ad with the revolving speed of 716 rps has been found. Further increase of the star (pulsar) mass and corresponding slowing-down of its rotation changes the star properties to the physical state called in astronomy as the white dwarf. The white dwarf circulation period is few hours. Continuous increase of the white dwarf mass transforms it into the red giant. In the concluding stage of the neutron state, the star, accumulating a corresponding mass, decreases significantly the rotation speed, and the density of outer layers (taking into account the corresponding decrease of the surface gravitation forces) also decreases by several orders of magnitude. It is the stage of the ordinary star. This stage is divided into the initial one (hot ordinary star) and the next (cold ordinary star). Then the star increases its mass up to that of the proto-star and decreases the rotation speed.

Specific physical properties of a star - the mass, temperature, luminosity, rotation speed, density, surface gravity force - correspond to each stage of its life. As the mass and the radius of a star increase, the surface moves away from the center, and the gravitation force on the outer layers and the surface substance density correspondingly decrease. However, inside the star, the star substance remains to exist, and it possesses the properties corresponding to the previous evolution stage of this star. Particularly, inside the ordinary cold star, on a certain depth, the substance in the hot star state is reserved; deeper - the matter is in the neutron state; and finally, in the center - the BH state. In all the layers of the star substance, the gravitation force grows in accordance with the principle and the formula of the vortex gravitation.

In modern cosmology, nova outbursts are treated as the end of the evolution of these stars.

From the viewpoint of vortex cosmogony, nova and super-nova outbursts should be treated as indications of new stars because: 
- outer layers of each star, or radio-, electromagnet, and light radiations, emitted by this star, are held back at its surface since the gravitation force at this surface exceeds the centrifugal forces acting due to the star rotation or the radiation momentum. Because the star volume and radius increase permanently, the vortex gravitation force on the surface of this star has to decrease inversely proportionally with square of the distance from the star (torsion) center. Therefore, evolution moments have to come in the life of each new star when the gravitation force on the object surface decreases down to the value incapable of holding back not only radiations, but also - outer layers of the star substance. In such cases, astronomy observations fix either the appearance of nova stars or super-nova outbursts accompanied by ejection of the star substance to the cosmic space. Further super-nova mass increase results in the rotation speed, and hence centrifugal force acting onto the outer layers, decrease. As a result, the star massive will be in a stable state, and the detachment of the outer layers into the cosmic space will over.

\subsection{CAUSE OF THE ELLIPSOIDAL SHAPE OF ORBITS}

It is known that the planets circulate around the Sun by an ellipse with a small eccentricity. This fact is accounted for from the viewpoint of vortex gravitation; moreover, it serves as a convincing proof of the existence of this gravitation with its discoid plane-symmetrical configuration (Section 2.2).

The cause of the planets orbit "compression" is the inclination of these orbits to the sun torsion plane. This statement is based on the following.

As is known, the planes of orbital motion of all the planets are situated with small deviations one from another. Consequently, planet orbit planes have inclinations to the plane of the sun gravitation torsion, where the highest gravitation force for this orbit acts, and the planets should intersect the sun torsion in two points during their orbital motion. As will be shown below, these intersection points coincide with the centers of perihelion and aphelion.

In the aphelion and perihelion, the sun gravitation force acts onto the planets with the highest magnitude at this orbit, and hence the orbit possesses a maximum curvature. At going out (deviation) from the sun torsion plane, the gravitation forces decrease and the planet trajectory "unbends" (Fig. 3). As such the cycle of the gravitation force and motion trajectory change repeats for each planet and for each turn around the Sun. The more the planet circulation trajectory is deviated from the central sun torsion plane, the higher is the degree of the gravitation force decrease in these regions, and hence the higher is the degree of "straightening" or "compression" of the orbit. Due to a permanent cyclic change of these forces, the orbit becomes ellipsoidal.

At significant inclinations and high speeds, the orbit of a satellite (meteorite, comet) have a hyperbola or parabola trajectory, and, correspondingly, the celestial object, once turning around the Sun, abandons the sun gravitation torsion field forever.

\section{Determining of the sun torsion direction}

On the basis of the stated above, it is obvious that the orbit trajectory eccentricity value of any planet depends on the value of inclination of this orbit to the sun torsion. Therefore, a reverse relation takes place, i.e. the lower the orbit eccentricity, the lower the inclination of the planet orbital plane to the sun torsion plane. Since the Venus orbit has the least eccentricity, for preliminary calculations, it is permissibly to accept the following property of the sun torsion:

- the direction of the sun gravitation torsion in the World coordinates coincides with the Venus orbital plane direction to a highest degree. Therefore, all the inclinations and latitudes of any astronomical point can be determined with regard to the orbital plane of Venus with a small correction up to 0.5 degree. 


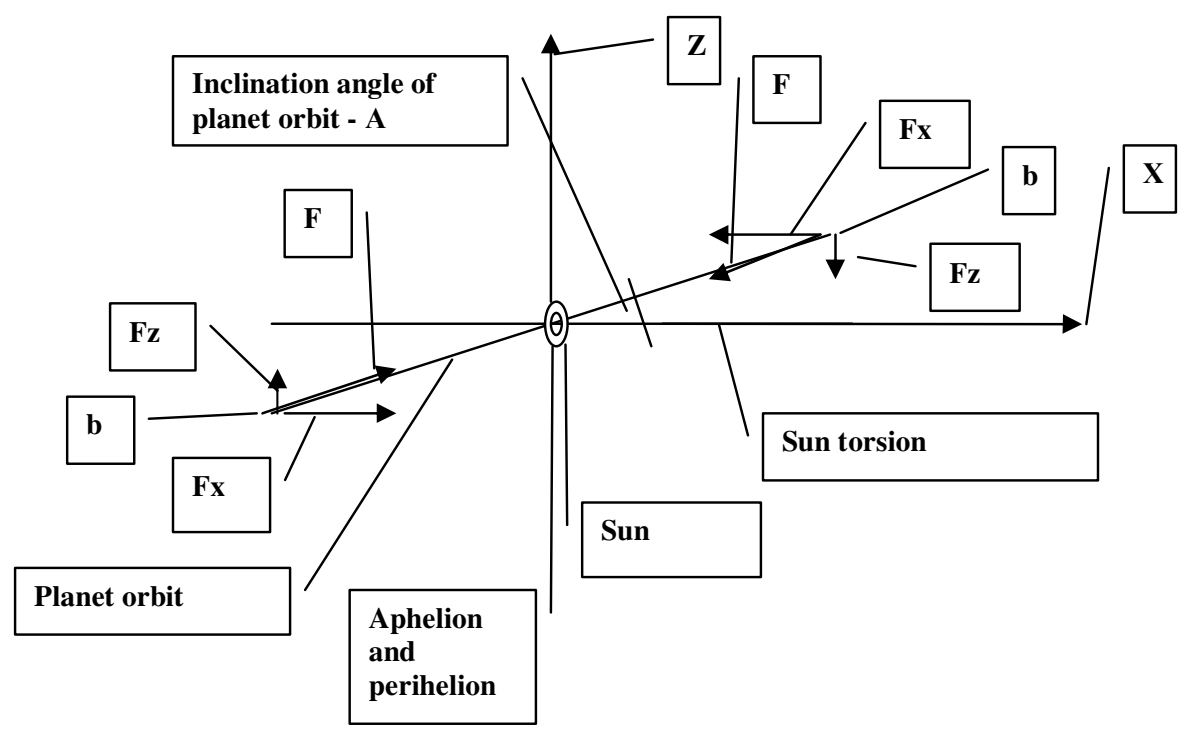

Fig. 3 Kinematical scheme of orbital motion

Let's consider the planet circulation in more detail with the Mercury motion as an example, in accordance with its heliocentric coordinates of 1993 [5].

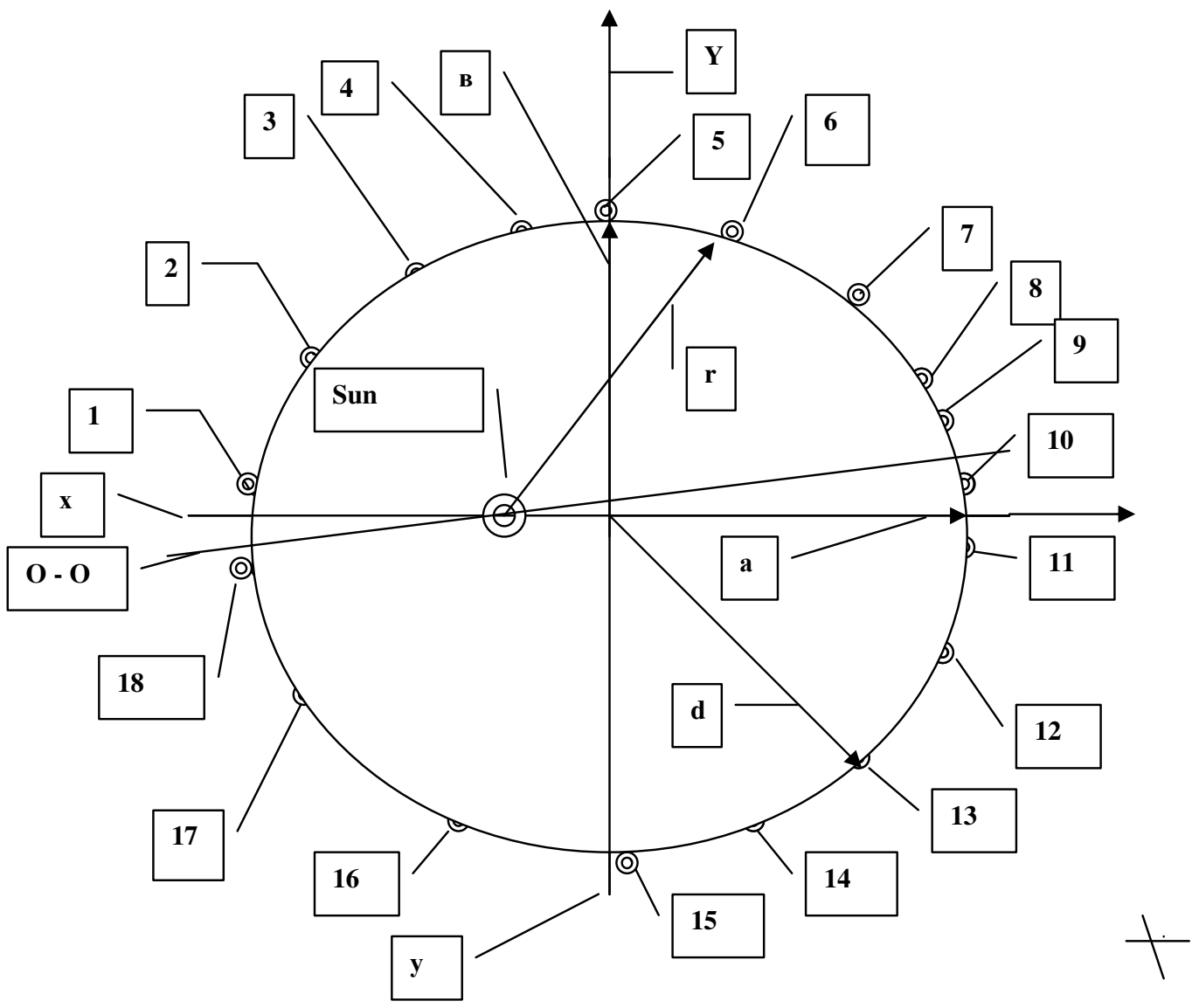

Fig. 4 Orbit of Mercury 
In Table $\mathbf{1}$ and Fig's $\mathbf{3}$ and $\mathbf{4}$, the following denotations are used:

$\mathbf{Z}$ - the torsion rotation axis

№ - numbers of the Mercury orbit points according to the astronomy calendar of 1993

Д - the heliocentric longitude $\mathrm{J} 2000.0$ of these points

$\mathbf{r}$ - the radius-vector, in $\mathrm{A}$. $\mathrm{U}$.

$\mathbf{d}$ - the distance from the ellipse center to the point under study, in million $\mathrm{km}$.

$\mathbf{V}$ - the orbital speed, in $\mathrm{km} / \mathrm{s}$

$\mathbf{R}$ - the curvature radius, in million $\mathrm{km}, \mathbf{R}=\mathbf{a}^{2} \mathbf{b}^{2} / \mathbf{d}^{3}$ where $\mathbf{a}, \mathbf{B}$ are the major and minor axes

Fc - the centrifugal forces

$\mathbf{F g}$ - the gravitation forces.

The values of centrifugal and gravitation forces are in portions of the planet mass.

$\mathbf{O}-\mathbf{O}$ - the apse line coinciding with the line of intersection of the Mercury orbital plane with sun torsion plane. The center of Mercury perihelion has the longitude of $(\mathbf{8 5 . 8 3}-\mathbf{8 . 1 8})$ degree regarding to the point №1 in 1993. On the basis of comparison of latitudes from the astronomy calendar, it has been established that the Mercury traverses the Sun (Venus) torsion in the aphelion and perihelion. The same is true for the other planets. Therefore, at these parts of the planet motion, the orbit curvatures are maximum and equal to each other, and the gravitation forces correspond to their classical values, i.e. they are inversely proportional to square of the distance to the Sun or are equal to the centrifugal forces.

Thus, the exact position of the sun torsion by its latitude relatively to the ecliptic is indicated by the latitudes of the aphelion and perihelion centers of each planet and by the apse line directions of these planets.

Comparing the astronomy point latitude values with the ratio of the gravitation and centrifugal forces in these space points, one can find that the more the planet orbit is inclined to the sun torsion, the higher is the difference between the Newtonian calculated gravitation forces and the actual centrifugal forces in those same points.

We consider two points of the Mercury orbit (№ 9 и № 10 in Fig. 3).

Table 1

$\begin{array}{clccccrr}\text { № } & \text { Д, degree } & \text { r } & \text { d } & \text { V } & \text { R } & \text { Fc } & \text { Fg } \\ 9 & \mathbf{2 5 0 , 0 4} & \mathbf{0 , 4 6 5 7} & \mathbf{5 8 , 1 1} & \mathbf{3 8 , 4 1} & \mathbf{5 5 , 4 6} & \mathbf{2 6 , 6 0} & \mathbf{2 7 , 3 2} \\ \mathbf{1 0} & \mathbf{2 6 3 , 7 8} & \mathbf{0 , 4 6 5 9} & \mathbf{5 8 , 0 3} & \mathbf{3 8 , 9 6} & \mathbf{5 5 , 6 9} & \mathbf{2 7 . 2 6} & \mathbf{2 7 , 2 9}\end{array}$

The distance between point 10 and the Sun is 0.4659 x $150=\mathbf{6 9 . 8 8 5}$ million $\mathbf{~ k m}$. For point 9 it is $\quad-0.4657$ x $150=\mathbf{6 9 . 8 5 5}$ million $\mathbf{~ k m}$.

The distance of point 10 from the Sun is 1.0004 times longer than that for point 9 . Therefore, in point 10, the Newtonian sun gravitation forces have to be 1.001 times less than those in point 9 (see Table 1). In reality, according to the calculation, the value of centrifugal forces in point 10 are 1.025 times higher as compared to point 9 which is associated with a larger orbit curvature in this point (see Table 1). Since the planet circulation centrifugal forces are reactive and always equal to the gravitation forces, it is follows from the above-said that, in this region of Mercury motion trajectory, the classical gravitation law is not fulfilled.

On the basis of the vortex gravitation model with a plane-symmetrical configuration, this paradox has a physical-mathematical ground. The Newton's world attraction law or formula 10 in Section 2.1 can describe the action of the gravitation forces only in the plane of the gravitation torsion. 
The above-presented calculation of the centrifugal forces appearing at the planet motion in the aphelion shows that the inertial circulation of the planets along an ellipsoidal trajectory in a central-symmetrical gravitation field is impossible in accordance with the classical ideas.

It should be noted that the planet orbit perihelion revolving round the Sun is also accounted for by a permanent change of the force magnitudes acting upon the planets.

\subsection{CALCULATION OF GRAVITATION IN THREE-DIMENSIONAL MODEL}

The change of the dynamical properties of the planets at their inclination, discussed in Section 3.3, gives a possibility to obtain a formula describing the change of gravitation forces in the three-dimensional model.

Comparing the orbit compression coefficients for all the planets with cosine of the angle of inclination of these orbits to the sun torsion, one finds that these values are directly proportional to each other:

\section{b/a $\sim \operatorname{Cos} A \quad(16)$}

\section{Proofs of equation (16)}

axis $\mathbf{X}$ - the direction of the parent torsion central plane.

axis $\mathbf{Z}$ - the rotation axis of the parent torsion.

A - the inclination angle of the satellite (planet) orbit torsion.

OB - the curvature radius of the torsion-satellite revolving at the coincidence of the satellitetorsion motion trajectory with the parent torsion rotation plane, i.e. at the perihelion or aphelion, or at the apex of the orbit major semiaxis:

$$
\mathbf{O B}=\mathbf{b}^{2} / \mathbf{a}
$$

OD1 - the curvature radius of the torsion-satellite revolving when it moves in a region possessing the inclination of angle $\mathbf{A}$ from the parent torsion central plane, i.e. at the apex of the orbit minor semiaxis:

$$
\text { OD1 }=\mathbf{a}^{2} / \mathbf{b}
$$

We prove that the equation $\cos \mathbf{A}=\mathbf{b} / \mathbf{a}$ is fulfilled at equalities (17) and (18)



Fig.5. Plane projections of minor and major orbital semiaxes. 


\section{Proof:}

First we draw a segment $\mathbf{O B}$ on the axis $\mathbf{X}$ (fig.5) coinciding with the apses line. This segment is to be equal to the curvature radius in the major semiaxis apex and is directed along the sun torsion central plane or the apses line.

Let's now draw a line from the center $\mathbf{O}$ with the angle $\mathbf{A}$; the direction of this line has to coincide with the minor semiaxis apex.

Since, from the problem condition, $\cos \mathbf{A}=\mathbf{b} / \mathbf{a}=\mathbf{O B} / \mathbf{O C}$, then:

$$
O C=O B a / b=\left(b^{2} / a\right)(a / b)=b
$$

Let's drop a perpendicular from point $\mathrm{C}$ on axis $\mathrm{X}$, as the angle $\mathbf{O C D} 2$ is right:

$$
\begin{aligned}
& \text { OC/OD } 2=\cos A=b / a, \text { whence } \\
& \text { OD } 2=\text { OC } \mathbf{a} / \mathbf{b}=\mathbf{b}(\mathbf{a} / \mathbf{b})=\mathbf{a},
\end{aligned}
$$

And finally we drop a perpendicular from point D2 on line OC, as the angle $\mathbf{D}_{1} \mathbf{D}_{2} \mathbf{O}$ is right:

$$
\text { OD2 } / \text { OD1 = cos A = b/a, whence OD1 = OD2 }(a / b)=\left(a^{2} / b\right)
$$

Therefore, equations (17) and (18) are fulfilled provided that $\cos A=\mathbf{b} / \mathbf{a}$. That is, the cosine of the planet orbit inclination angle in the minor semiaxis apex to the sun torsion plane is equal to the compression coefficient of this orbit.

Note 1. The inclination A of an orbital point does not coincide with the angle of inclination of this point indicated in astronomy calendars, because, according to the astronomy rules, all the coordinates in the Solar system are measured heliocentrically and from the ecliptic plane. Since the centrifugal forces are reactive and always equal to the sun attraction forces, these centrifugal forces may be considered as experimental or etalon values for the estimation of the accuracy and correctness of the results of gravitation forces calculations. Therefore, the change of the value of the planet centrifugal forces at a change of their coordinates is always equal to the change of the value of the gravitation force acting onto this planet.

\section{Determining of the three-dimensional gravitation coefficient $\mathrm{Kg}$.}

Let's write the formulas to determine the orbit (ellipse) curvature radius:

- in the major semiaxis apex or in perihelion and aphelion:

- in the minor semiaxis apex:

$$
\begin{aligned}
& \text { Rкр. } \mathbf{a}=\mathbf{b}^{2} / \mathbf{a} \\
& \text { Rкр.в }=\mathbf{a}^{2} / \mathbf{b}
\end{aligned}
$$

On the basis of the $2^{\text {nd }}$ Kepler law, the planets change the orbital velocity $(\mathbf{V})$ as a function of the distance to the Sun $(\mathbf{R})$, in the limits of their orbits, in the following proportion:

$$
\text { Va } \sim \text { 1/ Ra Vb } \sim \text { 1/ Rb (21) where }
$$

Va - the orbital speed in the perihelion (aphelion), i.e. in the apex of the planet orbit major semiaxis,

$\mathbf{V b}$ - the orbital speed in the apex of the planet orbit minor semiaxis

$\mathbf{R a}$ - the distance from the Sun to the aphelion (perihelion).

$\mathbf{R b}$ - the distance from the Sun to minor semiaxis apex.

The centrifugal force is determined from the formula:

$$
\mathbf{F c}=\mathbf{m} \mathbf{V}^{2} / \mathbf{R \kappa p}
$$


Substituting (19) - (21) into (22):

$$
\begin{aligned}
& \text { Fca }=m V^{2} / \text { Rкр. } a \sim m a / R^{2} b^{2}
\end{aligned}
$$

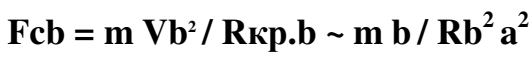

Since the gravitation forces in the aphelion and perihelion Fa correspond to their classical values or to the centrifugal forces, then, to determine a deviation of the gravitation forces in the torsion periphery (in the minor semiaxis apex - point b), it is necessary to determine the analogous deviation of the values of the centrifugal forces as compared to those same forces in the perihelion. For this purpose, we divide formula (24) by formula (23):

$$
\mathbf{F c b} / \mathbf{F c a}=\left[\mathbf{b}^{3} / \mathbf{a}^{3}\right]\left[\mathbf{R a}^{2} / \mathbf{R b}^{2}\right]
$$

Here the relative value $\mathbf{R a}^{2} / \mathbf{R b}^{2}$, in accordance with formula $\mathbf{1 0}$ in Section $\mathbf{2}$ or with the Newton formula, determines the gravitation force change as a function of the change of the distance from the torsion center to the points under consideration.

According to the expression (16), the value b/a equals to the cosine of the inclination angle in the considered point. Hence, this value determines the change of the gravitation forces as a function of the inclination of the considered point to the sun torsion.

Therefore, one can write:

$$
\mathbf{b}^{3} / \mathbf{a}^{3}=\operatorname{Cos}^{3} \mathrm{~A}=\mathrm{Kg}
$$

The gravitation forces in any point of the cosmic space are determined by the formula:

$$
\mathrm{Fv}=\mathrm{Fg} \operatorname{Cos}^{3} \mathrm{~A} \text {, (26) where }
$$

Fg - the gravitation force in the two-dimensional model (formula 10 in Section 2 or Newton equation)

Fv - the gravitation force in the three-dimensional model

Consequently, using the gravitation coefficient $\mathrm{Kg}$, one can determine the gravitation forces in any point distant from the center of a cosmic torsion.

Formula (26) shows that, when moving away from the gravitation torsion plane, parallel to the torsion axis, the gravitation force decrease inversely as the cube of the distance $-\mathbf{1} / \mathbf{s}^{\mathbf{3}}$

\section{EXPERIMENTAL VERIFICATION OF THE VORTEX GRAVITATION EQUIATION -} (26)

Any theory is considered to be proved if its conclusions and formulas correspond to experimental facts. Since the gravitation forces correspond to the centrifugal (experimental) forces, then, to determine a deviation of the gravitation forces in the torsion periphery (in the minor semiaxis apex - point $\mathbf{b}$ ), it is necessary to determine the analogous deviation of the values of the centrifugal (experimental) forces as compared to those same forces in the perihelion.

\section{Pluto}

$\mathrm{a}=5906,375 \times 10^{6} \mathrm{~km}-$ major semiaxis, $\mathrm{b}=5720,32 \times 10^{6} \mathrm{~km}-$ minor semiaxis

$\mathrm{r}=5907,963 \times 10^{6} \mathrm{~km}-$ the distance from the Sun to the Pluto orbit minor semiaxis apex 
$\mathrm{Kc}-$ compression coefficient of the orbit

$\mathrm{Kc}=1-\mathrm{e}^{2}=\mathrm{b} / \mathrm{a}=\operatorname{Cos} \mathrm{A}=0,9685$

$\mathrm{Kg}$ - gravitation coefficient

$\mathrm{Kg}=\mathrm{b}^{3} / \mathrm{a}^{3}=\operatorname{Cos}^{3} \mathrm{~A}=0,9084$

$\mathrm{Rb}$ - curvature radius in the Pluto orbit minor semiaxis apex

$\mathrm{Rb}=\mathrm{a}^{2} / \mathrm{b}=6098,48 \times 10^{6} \mathrm{~km}$

$\mathrm{Vb}=4,581 \mathrm{~km} / \mathrm{c}$ - the orbital speed in the apex of the Pluto orbit minor semiaxis

The centrifugal force in the minor semiaxis apex

$\mathbf{F c b}=\mathbf{0 , 0 0 3 4 4}$ Mp, Mp - mass of the Pluto.

Newtonian gravitation forces in its point

Fgb $=0,00382 \mathrm{Mp}(+11,1 \%$ concerning Fcb)

The vortex gravitation forces

$\mathrm{Fvb}=\mathrm{Fg} \times \mathrm{Kg}=0,00382 \times 0,9084=0,00347 \mathrm{MII}(+0,87 \%$ concerning Fcb $)$

2. Mercury (analogous calculation)

$\mathrm{a}=57,91 \times 10^{6}$, в $=56,67 \times 10^{6}, \mathrm{r}=58,395 \times 10^{6}$.

$\mathrm{e}=0,2056$, Ксж $=\mathrm{B} / \mathrm{a}=1-\mathrm{e}^{2}=\operatorname{Cos} \mathrm{A}=0,9786, \mathrm{Kg}=0,9372$.

$\mathrm{Rb}=59,177, \mathrm{Vb}=46,4775$

The centrifugal force in the minor semiaxis apex

Fcb = 36,503 Mm, Mm - mass Mercury

Newtonian gravitation forces

Fgb $=39,09 \mathrm{Mm},(+7,1 \%$ concerning Fcb $)$

The vortex gravitation forces

Fvb $=36,63 \mathrm{Mm}(+0,35 \%$ concerning Fcb $)$

Consequently, formula (9) is really correct.

For a more clear presentation of the influence of $\mathbf{K g}$ on the (classical) gravitation forces calculated in the plane model, we draw the epures of these forces acting on the periphery plane 
of the parent torsion which is situated parallel to the parent torsion central plane with the torsion rotation axis coordinate being $\mathbf{Z}=\mathbf{1}$ a. $\mathbf{u}$. (Fig. $\mathbf{6})$

Let's consider a torsion with the radius $\mathbf{R}=\mathbf{1 0} \mathbf{a}$. $\mathbf{u}$. (arbitrary units)

Epure 1 - gravitation forces Fn corresponding to the calculation in the two-dimensional model (formula $\mathbf{1 0}$ in Section $\mathbf{2}$ or the Newton formula)

Epure 2 - gravitation forces $\mathbf{F o}=\operatorname{Cos}^{3} \mathbf{A}$ Fn corresponding to the calculation in the threedimensional model (with the correction $\mathbf{K g}$ )

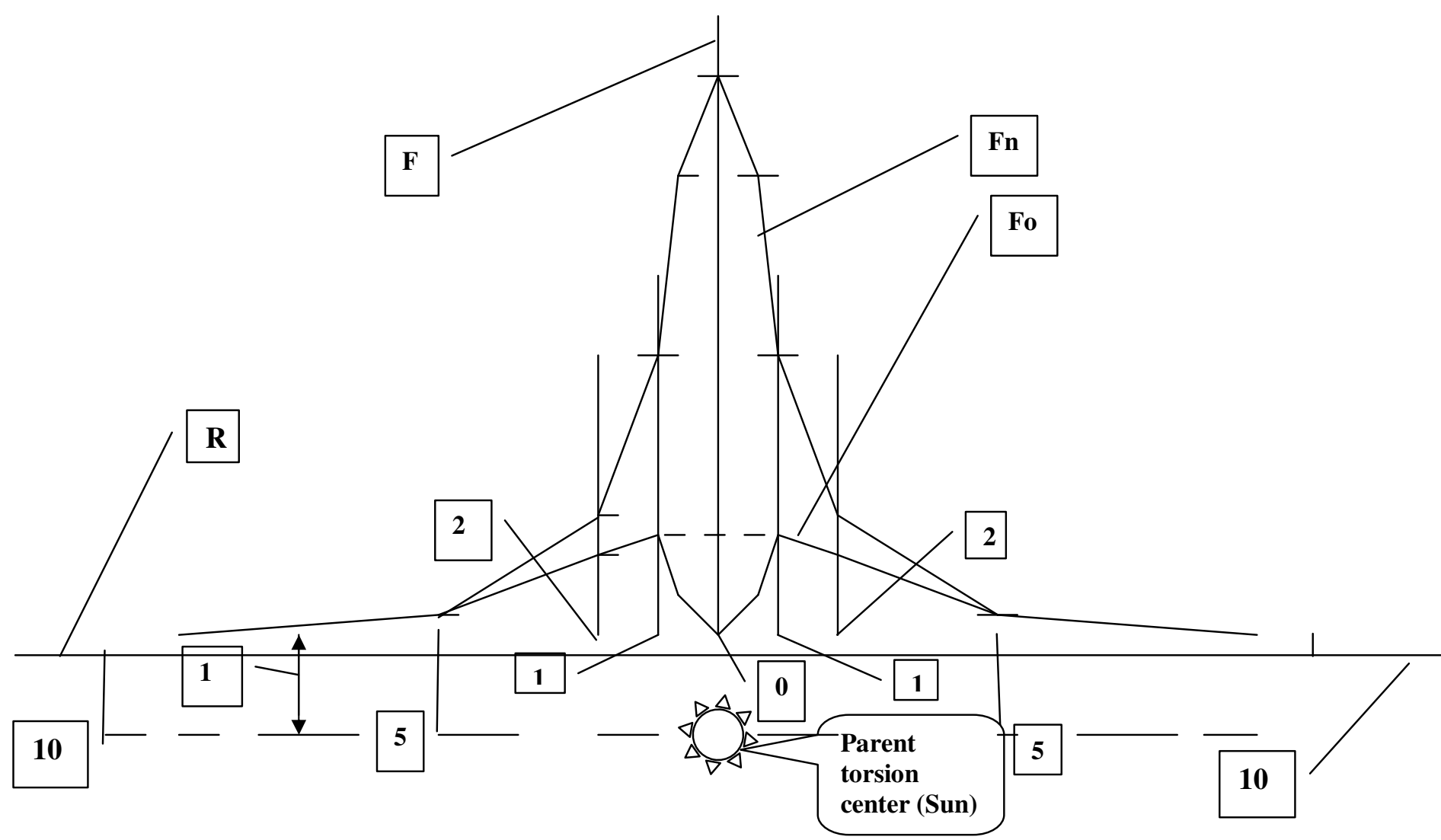

Fig. 6. Epures of the gravitation forces on the torsion periphery with $\mathrm{Z}=1$

The results of calculation of the gravitation forces, using the proposed scheme, have an essential difference for the space points with the inclination of their coordinates to the cosmic torsion plane over 10 degrees. On the periphery torsion regions close to the rotation axis, the gravitation forces should sharply rise according to the classical calculation, whilst, according to the vortex gravitation calculation, these forces tend to zero.

At the torsion front regions, $A \sim$ from -45 to +45 degree, it is necessary to take into account the corresponding front ether turbulence creating additional gravitation forces which will even its gradient. However, in any case, the graph of the gravitation forces in these zones will demonstrate either a constant value or a decrease of the gravitation forces. It is known that, according to the two-dimension gravitation formula (i.e. the Newton's one or formula 10 from Section 2), the gravitation (gravity) force on the pole is $\mathbf{F p}=$ $\mathbf{9 , 8 7} \mathbf{m}$. On the other hand, the actual gravity force determined by means of geodetic gravimetry is $\mathbf{F p}=\mathbf{9 , 8 3 m}$, which is $\mathbf{0 . 4 3 \%}$ less than the calculated value. 
This difference can be explained only on the basis of the above-indicated calculation in the frameworks of the three-dimensional gravitation model. That is, on the poles, the gravitation is created by the ether front turbulences, and the ether velocity is varied by a different law as compared to that in the longitudinal torsion direction. Hence, the gravitation force decreases differently in the longitudinal direction and in the pole direction. The gravitation force distribution in some cosmic torsion determines the form of a cosmic object created in this torsion. The configuration of epure № 2 is especially close to the galaxy configuration. Therefore, this calculation proves additionally a plane-symmetric configuration of the attraction forces. Also, the cosmic expedition of the automatic station to Venus in April 2006 has found the zone of small satellites revolving in one plane, and atmospheric craters have been found on the planet poles. The planet of Saturn is also surrounded by a ring of small satellites situated in one orbital plane. These facts convincingly prove the plane configuration of the planetary gravitation torsions, which is in agreement with the above calculations. The projecting of middleand long-distance cosmic flights with the taking into account the plane-symmetric gravitation torsion configuration will allow a significant reduction of the transport expenses.

\subsection{MOTION OF TORSION-SATELLITES}

In order to account for the motion of satellite torsions around the parent torsion center, it is sufficient to use the momentum conservation law and the property of a celestial body to increase its mass due to the own gravitation.

Let's write the angular momentum conservation law of motion:

\section{V R = const whence $\mathbf{K m ~ K v ~ K r = 1 ~ ( 2 7 ) , ~ w h e r e ~}$}

$\mathbf{M}, \mathbf{V}, \mathbf{R}$ - the mass, orbital velocity, and the distance to the planet circulation orbit.

$\mathbf{K r}, \mathbf{v}, \mathbf{m}$ - coefficients of the change of the celestial object orbital distance, velocity, and mass. The plant mass increase of $1.6 \times 10^{15} \mathrm{~kg}$ per year, given in [1], in a cosmic scale, is an alternating value and depends on the planet distance from sun torsion center because: - the cosmic dust density in any cosmic torsion, including the sun's one, increases from the periphery to the center. This is explained by the fact that the dust from outside, during its radial motion toward the center of the sun torsion under the action of gravitation, should remove into a lower volume, and hence it should become denser. The volume of each orbital layer with a negligibly small thickness is proportional to the area of this orbital surface (S) or to square of the distance from the orbit center:

$$
S=4 \Pi \mathbf{R}^{2} .
$$

Therefore, the cosmic dust density in any torsion increases as square of distance in direction toward the torsion center. Since the influx of cosmic dust into the planetary torsion is proportional to the density of the adjoining cosmic substance, one may arrive at a conclusion that the closer the torsion-satellite revolving orbit is to the parent torsion center, the higher the cosmic dust influx enters into the torsion of this satellite, which can be written as:

$$
\mathrm{Kr}=\mathrm{Km}-2 \quad(28)
$$

Substituting (28) to (27), one can find that:

$$
\mathrm{Km}=\mathrm{Kv} \quad \text { or } \mathrm{Kr}=\mathrm{Kv}-2
$$

Formulas $(28,29)$ prove:

1. 3rd Kepler law, because formula (29) is equivalent to this law:

$$
\mathrm{R} \sim \mathrm{V}-2 \text { or } \mathrm{V} 2 \mathrm{R}=\text { const }
$$


2. All the torsion-satellites move with an acceleration and by a spiral to the parent torsion center, because it follows from formulas (27),(28) and (29) that as the torsion satellite mass increases (at $\mathrm{Km}$ above zero), the distance to the torsion rotation center decreases, and its orbital speed increases. It should be noted that formulas 27,28,29 describe the satellite motion only in the central plane of the gravitation torsion. At inclination of the orbits of the celestial objectssatellites, the following motion trajectory is feasible:- at the initial stage of the existence of the torsion-satellite, a maximum increase of its relative mass occurs. Therefore, on the basis of the present section (form. 28), a rapid decrease of the orbital motion radius of this torsion occurs. As the satellite mass increases, the relative mass growth $(\mathrm{Km})$ decreases, because the absolute mass growth of the torsion-satellite is constant. After the accumulation of a considerable mass of the satellite substance, the relative increase of its mass should be negligible. Then the celestial objects-satellites (stars, planets, planet satellites), possessing the inclinations of their own orbits to the gravitation parent torsion disk plane, can stop the spiral approaching toward the parent torsion center and, afterwards, continue their spiral orbital motion, but toward the periphery, not to the center. This is accounted for by the fact that, during the orbital motion of the satellites in the minor semi-axis apex, i.e. in the region of a maximum deviation from the parent torsion plane, the centrifugal forces can be higher than the gravitation forces, which must cause an orbit radius increase of these satellites.

Let's consider the orbital motion of the Moon, which has the following characteristics:

- the orbit compression coefficient $\mathrm{Kc}=0.997$

- gravitational coefficient $\mathrm{Kg}=\mathrm{Kc} 3=0.991$

- Moon orbital speed in the minor semi-axis apex $\mathrm{Vb}=1.01858 \mathrm{~km} / \mathrm{s}$

- distance from the Earth to the minor semi-axis apex $\mathrm{Rb}=0.38378 \times 106 \mathrm{~km}$

- orbit curvature radius in the minor semi-axis apex Rкp $=0.3856 \times 106 \mathrm{~km}$

The centrifugal force acting on the Moon in the minor semi-axis apex is therefore:

$\mathrm{Fc}=2.691 \mathrm{Mm}$, where $\mathrm{Mm}$ is the Moon mass.

According to the Newton law, the Earth gravitation force acting on the Moon in the same orbital point must be:

$$
\mathrm{Fgn}=2.706 \mathrm{Mm}(+0.55 \%)
$$

In the model of vortex gravitation this force is:

$$
\mathrm{Fgv}=\mathrm{Fgn} \times \mathrm{Kc} 3=2.706 \times 0,991=2.682 \mathrm{Mm}(-0.33 \%)
$$

Therefore, from the Newton law, the Moon must approach to the Earth, and from the vortex gravitation model, it must move away from the Earth.Actually, the Moon orbit indeed moves away from the Earth by $38 \mathrm{~mm}$ a year; this fact additionally confirms the validity of the vortex gravitation model and, correspondingly, the incorrectness of the Newton law. Analogous calculations can be made in order to find the exact changes of the orbits of all the planets or stars. 


\subsection{MOONFLIGHT}

Let's consider a problem of comparing the works expended on getting over the gravitation attraction forces $(\mathrm{F})$ by a body, when traveling from point $\mathrm{A}$ to point $\mathrm{C}$ (see Fig.7) by the paths $\mathrm{AC}$ and $\mathrm{ABC}$ at two different $\mathrm{F}(\mathrm{r}, \varphi)$ dependences.



Fig.7. Scheme

In the first case, $\mathrm{F}$ is independent of $\varphi$ and obeys the Newton law (1)

$$
F(r)=G \frac{m_{1} \cdot m_{2}}{r^{2}},
$$

where $\mathrm{m}_{1}$ and $\mathrm{m}_{2}$ are the masses of bodies, $\mathrm{G}-$ the gravitation constant, and $\mathrm{r}-$ the distance between the bodies.

In the second case, F depends on $\varphi$ in accordance with formula (26) of Section 3.4

$$
\mathrm{F}(\mathrm{r}, \varphi)=\mathrm{G} \frac{\mathrm{m}_{1} \cdot \mathrm{m}_{2}}{\mathrm{r}^{2}} \cdot \cos ^{3}(\varphi)
$$

where $\varphi$ is the angle between axis OC and the position radius-vector of the replaced body.

As is known, the work equals to the path integral

$$
A=\int \vec{F} \cdot d \vec{r}
$$

Let $\mathrm{A}_{\mathrm{AC}}$ be the work expended at the transference $\mathrm{AC}$ for the case of the dependence (1). We determine the works $A_{A B}$ and $A_{B C}$. For $A^{\prime}{ }_{A C}$ being the work expended at the transference $A C$ for the case of the dependence (2) we determine, respectively, the works $\mathrm{A}^{\prime}{ }_{\mathrm{AB}}$ and $\mathrm{A}_{\mathrm{BC}}$.

Now we write the integral (3) for each case

$$
\mathrm{A}_{\mathrm{AC}}=\int_{\mathrm{r}_{1}}^{\mathrm{r}_{2}} \mathrm{G} \cdot \frac{\mathrm{m}_{1} \cdot \mathrm{m}_{2}}{\mathrm{r}^{2}} \mathrm{dr}
$$




$$
\begin{aligned}
& \mathrm{A}_{A B}=\int_{0}^{\varphi_{B O C}} \mathrm{G} \cdot \frac{\mathrm{m}_{1} \cdot \mathrm{m}_{2} \cdot(\cos (\varphi)-\sin (\varphi)) \cdot \cos \left(\frac{3 \cdot \pi}{4}+\varphi\right)}{\mathrm{r}_{1} \cdot \sin \left(\frac{\pi}{4}-\varphi\right)} d \varphi \\
& \mathrm{A}_{\mathrm{B} C}=\int_{0}^{\varphi_{B O C}} \mathrm{G} \cdot \frac{\mathrm{m}_{1} \cdot \mathrm{m}_{2} \cdot(\cos (\varphi)+\sin (\varphi)) \cdot \cos \left(\frac{\pi}{4}+\varphi\right)}{\mathrm{r}_{2} \cdot \cos \left(\frac{\pi}{4}-\varphi\right)} d \varphi \\
& \mathrm{A}_{A B}^{\prime}=\int_{0}^{\varphi_{B O C}} \mathrm{G} \cdot \frac{\mathrm{m}_{1} \cdot \mathrm{m}_{2} \cdot(\cos (\varphi)-\sin (\varphi)) \cdot \cos \left(\frac{3 \cdot \pi}{4}+\varphi\right) \cdot \cos ^{3}(\varphi)}{\mathrm{A}_{\mathrm{AC}},} d \varphi \\
& \mathrm{A}_{\mathrm{B} C}^{\prime}=\int_{0}^{\mathrm{r}_{1} \cdot \sin \left(\frac{\pi}{4}-\varphi\right)} \mathrm{G} \cdot \frac{\mathrm{m}_{1} \cdot \mathrm{m}_{2} \cdot(\cos (\varphi)+\sin (\varphi)) \cdot \cos \left(\frac{\pi}{4}+\varphi\right) \cdot \cos ^{3}(\varphi)}{\mathrm{r}_{2} \cdot \cos \left(\frac{\pi}{4}-\varphi\right)} d \varphi
\end{aligned}
$$

where $\mathrm{r}_{1}-$ the distance OA, $\mathrm{r}_{2}-\mathrm{OC}$, and $\varphi_{\mathrm{BOC}}-$ the angle BOC.

Formula (36) is valid because, in this direction, the forces (30) and (31) are equal to each other. Calculating the integrals (36-38) numerically for the case of moonflight $\left(r_{1}=6400 \cdot 10^{3} \mathrm{~m}, \mathrm{r}_{2}=\right.$ $40000000 \mathrm{~m}, \mathrm{~m}_{2}=6 \cdot 10^{24} \mathrm{~kg}, \mathrm{~m}_{1}=1 \mathrm{~kg}$ ), one obtains $\mathrm{A}_{\mathrm{AC}}=6.1554643 \cdot 10^{7} \mathrm{~J}, \mathrm{~A}_{\mathrm{AB}}=6.1140242$ $\cdot 10^{7} \mathrm{~J}, \mathrm{~A}_{\mathrm{BC}}=4.1440045 \cdot 10^{4} \mathrm{~J}, \mathrm{~A}^{\prime} \mathrm{AB}=4.5279719 \cdot 10^{7} \mathrm{~J}, \mathrm{~A}^{\prime}{ }_{\mathrm{BC}}=3.5727542 \cdot 10^{5} \mathrm{~J}$.

One can see that $A_{A C}=A_{A B}+A_{B C}$, which just must be the case for the Newtonian forces when the work does not depend on the transference path from point $\mathrm{A}$ to point $\mathrm{C}$.

In the case of the law (31), the work on the path $\mathrm{ABC}$ equals to $\mathrm{A}^{\prime}{ }_{\mathrm{ABC}}=\mathrm{A}^{\prime}{ }_{\mathrm{AB}}+\mathrm{A}^{\prime}{ }_{\mathrm{BC}}=4.5636994$ $\cdot 10^{7} \mathrm{~J}$. This is less than the work $\mathrm{A}^{\prime}{ }_{\mathrm{AC}}=\mathrm{A}_{\mathrm{AC}}=6.1554643 \cdot 10^{7} \mathrm{~J}$.

The ratio (decrease) of the works is $s=\mathbf{A}^{\prime}{ }_{\mathbf{A B C}} / \mathbf{A}_{\mathbf{A C}}=\mathbf{0 . 7 4 1 4 0 6 2}$. The value of $s$ depends on the distances $r_{1}$ and $r_{2}$ and on the transference path. Thus, the transference by the path ABC in the case of the law (2) is more energetically preferable than that directly by the path AC. The above calculation shows that the moonflight with a detour of the Earth torsion should decrease the fuel consumption on $25 \%$.

At present, most interplanetary cosmic apparatus get accelerations which can not be explained on the basis of cosmic calculations in the relativity theory of Einstein. Particularly, deviations have 
been found for the apparatus of «Galileo», «Rosetta» and «Cassini». The suggested model of vortex gravitation (formula 26) shows that, if the trajectory of the satellite flight does not coincide with the Sun gravitation torsion plane, then one should take into account the value of gravitation coefficient in the calculation of solar gravity acting onto the satellites. This coefficient $(\operatorname{Cos} 3 \mathrm{~A})$ reduces the value of solar gravity, which gives a certain acceleration to cosmic satellites and results in a deviation of the motion trajectory.

\subsection{CAUSES OF EBBS AND FLOWS}

It is known that the appearance of the sea ebbs and flows twice in 24 hours and 50 minutes is explained by the influence of the gravitation fields of the Moon and Sun and by the centrifugal forces (Galileo, Descartes, Newton and others).

Since the Moon and Sun are in zenith under one Earth surface point only once a day (or in 24 hours $50 \mathrm{~min}$.), they could not have an equal influence upon this point by means of their gravitation twice a day. The model of vortex gravitation gives a new explanation of this phenomenon, which consists in the following.

On the basis of the vortex gravitation, the form of the Earth gravitation torsion is discoid and plane-symmetrical. Its direction mainly coincides with the Moon orbit plane which is inclined to the ecliptic on 5 degrees and $9 \mathrm{~min}$. Meanwhile, the Earth equator plane has the inclination to the ecliptic of 23.7 degree. The compression coefficient of the Moon orbit, or the cosine of the inclination, is 0.9985. That is, the inclination of the Moon orbit to the Earth torsion is 3 degree and 6 min (Section3.4, formula (1)). A geometrical comparison of these inclinations evidences that each Earth surface point rotates with a considerable angle as related to the Earth gravitation torsion plane. Thus, one and the same point of the Earth surface every time intersects the gravitation torsion across its direction, or it first approaches to and then moves away from the torsion. The force of the vortex Earth gravitation, acting onto this point, changes in this case correspondingly (see Fig. 8).

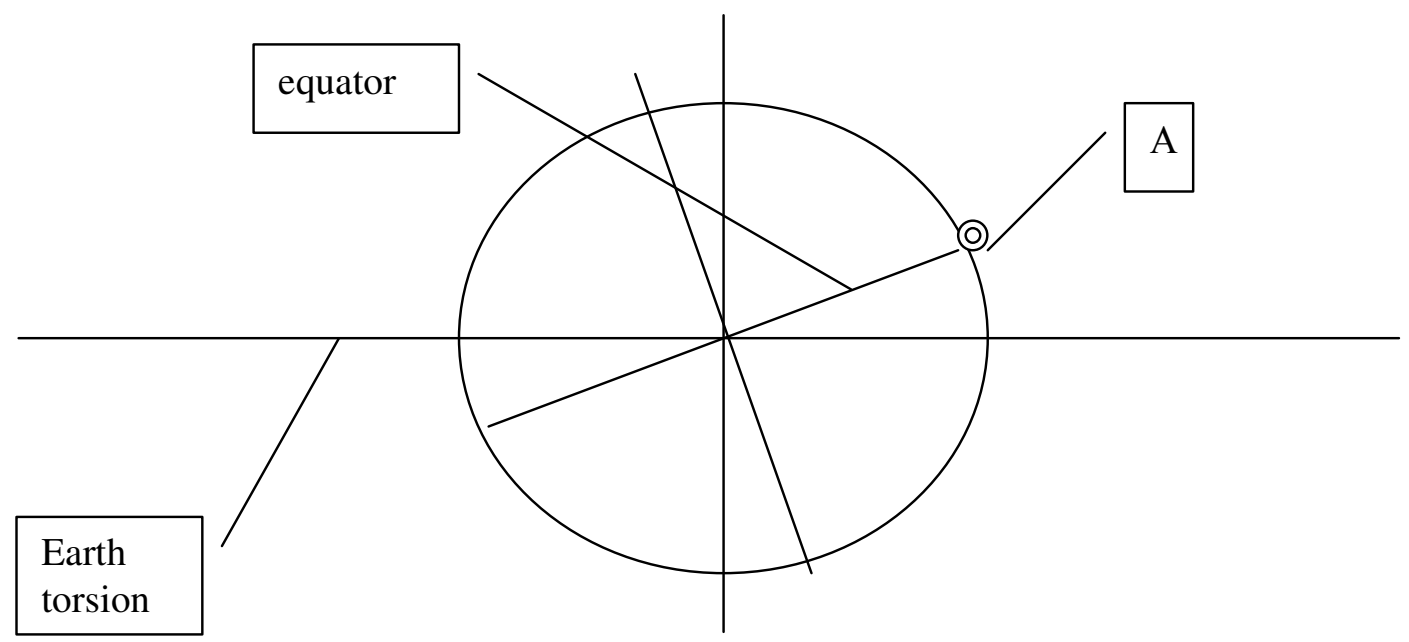

Fig. 8 Inclination of the Earth equator 
Point A, at its daily motion around the Earth center, must twice intersect the Earth torsion central plane, and it must twice move away from it. Consequently, the Earth gravitation forces achieve their maximum influence on point A twice a day, and they achieve their minimum value also twice a day. This has a physical influence upon the Earth surface and the level of water in this point. This, in turn, accounts for the fact that the ebbs and flows occur in one point twice a day. As was mentioned in Section 3.5, by geodetic gravimetry, it has been established that the Earth gravitation force on the poles has a $0.43 \%$ lower value as compared with the theoretical calculation. This confirms the above-noticed non-uniform gravitation force on the Earth surface.

As is known, the sun gravitation force on the Earth orbit is $0.06 \%$ of the gravitation force of our planet on its own surface. The Moon affects on the Earth surface with the attraction force of $0.0003 \%$ of the Earth gravitation force.

When comparing these values, it is obvious that the wave-like change of the Earth gravitation forces under the action of the own rotation occurs, at the Earth surface, with a much higher intensity than the same change of the Earth gravitation under the action of the gravitation of the Sun and Moon at their mutual rotation.It should be noted that a permanent wave-like change of the gravitation field intensity in the Earth surface layers could be a noticeable catalyst for the tectonic motions of the earth's crust plates and seismicity increase. Also, the cyclic change of the gravitation force in one point should be taken into account in precision investigation and productions in medicine and other areas of natural sciences, including the registration of sport records.

\subsection{MAGNETIC POLE OF THE EARTH}

On the basis of the vortex gravitation, one can suppose that the magnetic field of the Earth is due to the rotation of the Earth ether torsion, and it is not due to the merely Earth rotation itself. Because the Earth rotation axis $\left(\mathrm{O}_{3}\right.$, Fig. 8) is inclined to the Earth torsion rotation axis $\left(\mathrm{O}_{0}\right)$ on the angle of $\sim 20$ grades, one can arrive at a conclusion that the Earth surface intersects the Earth torsion and magnetic field axis in latitude of $\sim 70$ grade. Taking into account the Earth rotation, it is obvious that the Earth magnetic poles accomplish the daily revolution around the geographic poles in the same latitudes (70 degree). The attitudes of the magnetic poles depend on the time of day. This accounts for the convergence of magnetic and geographic poles and the instability of the Earth electromagnetic orientation.

\subsection{TUNGUS METEORITE}

The fall of the meteorite in 1908 near the river of Tungus in Siberia has put a number of questions, and some of them are not decided so far. Particularly, neither a shell-hole nor traces of the meteorite substance have been found, which contradicts to physics laws. The model of vortex gravitation allows an analytical explanation of this paradox.

It is supposed that the Tungus meteorite was a new-created torsion-satellite of the Earth torsion which formed almost in the center of the front (peripheral) region of the Earth torsion. This is supported by the value of the Tungus latitude (60 degree), while the Earth torsion axis latitude is 70 degree (see Section 3.9). Therefore, this torsion-satellite formed in the periphery region with a deviation of $\sim 10$ degree from the Earth torsion rotation axis. In this region, the vortex gravitation force is considerably higher than that in the more distant periphery regions (i.e. regions farthest from the Earth torsion axis). In accordance with the predominance of the gravitation forces under the centrifugal ones, the tungus torsion (after its appearance) did not form as a perpetual satellite of the Earth, but it directed toward the Earth center. This torsion had not time to accumulate the cosmic substance and was in the state of a Black Hole or a pulsar. During collision with the Earth surface, the commonly-known colossal explosion occurred without an emission of the meteorite substance, which the tungus torsion did not contain (and even if it would contain, the quantity of this substance was negligible; and also this substance 
represented micro-particles like nucleons). The flow of micro-particles of such sizes could not create any visual traces on the Earth surface and hence it was not found. The damage traces in the fall place were produced only by sadden ultra-low pressure release into the atmosphere which caused a destructive air wave running few times around the Earth.

\subsection{EXPANSION OR COMPRESSION OF THE UNIVERSE?!}

At present, the moving of galaxies away from each other is accounted for by the expansion of the Universe which began due to the so-called "Big Bang".

For the analysis of the galaxies' moving away, we use the following known physical properties and laws:

1. The galaxies revolve round the center of the metagalaxy making one turn per $\mathbf{1 0 0}$ trillion years [4].

Therefore, the metagalaxy is a giant torsion where the laws of vortex gravitation and classical mechanics are valid (Section 3.2).

2. Because Earth increases its mass [1], one may assume that all the other celestial objects and their systems (galaxies) also increase the masses under the action of their own gravitation in accordance with the laws presented in Section 3.2. In this case, on the basis of the formulas of this section, it is evident that the galaxies must move by a spiral toward the metagalaxy center with an acceleration which should be inversely proportional to the distance from the metagalaxy center or to the galaxy mass increase.

The radial acceleration of the galaxies during their motion toward the metagalaxy center results in their mutual moving away from each other, which has been indicated by Hubble, and which, by now, is mistakenly treated as the expansion of the Universe.

Thus, the following conclusion can be made from the above-said:

- The Universe does not expand, but, on the contrary, twists by a spiral or contracts.

It is quite probably that a metagalaxy Black Hole is situated in the center of the metagalaxy, and it is impossible therefore to observe it.

At the circulation of the galaxies around the metagalaxy center on a lower orbit, the orbital motion speed of these galaxies must be higher than that of the galaxies moving on a higher orbit. In this case, the galaxies must close together in certain time mega-intervals. This explains the approaching of the galaxy M31.

In the initial stage of the cosmic torsion appearance, it should be in the state of $\mathrm{BH}$ (see Section 3.1). In this period, the increase of the cosmic torsion relative mass is maximal. Therefore, the magnitude and vector of the velocity of this torsion $(\mathrm{BH})$ also possess maximum changes. That is, the character of motion of the Black Holes does not correspond to the motion of neighbor cosmic objects.

At present, the $\mathrm{BH}$ approaching to us has been found. The motion of this $\mathrm{BH}$ is explained by the above-discussed dependence. At considerable inclinations of the celestial object orbits to the plane of the corresponding torsion, an increase of the orbit radii of these objects or their systems can occur, or the stationary value of the radius will be conserved.

One should note the contradictions of the hypothesis of "Big Bang", which, by incomprehensible reasons, are not taken into account by modern science:

- according to the $2^{\text {nd }}$ law of thermodynamics, a system (Universe) being left to itself (after the explosion), turns into a chaos and disorder.

In reality, the harmony and order observed in the Universe contradict this law.

- any particle of the substance exploded with a huge intensity will acquire only straight radial direction of motion. Universal rotation of all the celestial objects and their systems in the space around the center or another object, including the metagalaxy, completely refutes the inertial nature of the motion of cosmic objects, obtained from the explosion. 
- according to the existing models (model of Fridman), the cause of the Big Bang was the compression of the Universe down to the size of the solar system. As the result of this supergiant densification of the cosmic substance, the Big Bang occurred. The followers of the idea of Big Bang pass over the absurdity of this hypothesis in silence, viz. - how could the infinite Universe be compressed and go into a limited volume of the size of the solar system!?

\subsection{VORTEX COSMOGONY}

It is obvious from the model of vortex gravitation that the main source of the origin and motion of celestial objects in the Universe is the vortex rotation of the cosmic ether which creates the vortex gravitation. The latter, in turn, at the moment of its appearance, gives a unique property to the torsion, namely - the ability to draw in any cosmic dust, which forms a celestial object at certain stages of the existence of this torsion.

\section{Principal scheme of vortex cosmogony.}

Since it was shown in Section 3.2 that all the cosmic torsions and (or) celestial objects-satellites move by a spiral toward the parent torsion center, it is straightforward to arrive at the following conclusion:

\section{- all the torsion-satellites were created in periphery sides of the parent torsions.}

The parent torsion formed in the periphery of the grand-parent torsion of a higher order, etc. Formation of torsion-satellites in the periphery of a parent torsion is accounted for by the fact that the ether vortex rotation weakens significantly in these layers of a parent torsion, and, in addition, the intersection of ether flows from two or more neighbor parent torsions are feasible there. These circumstances, on the basis of the hydro- aerodynamics laws, provide favorable conditions for the turbulence arising and, hence, for the appearance of local vortexes. Thus, the following scheme of the celestial objects and torsions creation can be proposed:

- satellite torsions formed in the periphery of planet torsions; planet torsions formed in the periphery of star torsions; star torsions - in the periphery of galaxy torsions; the latter - in the periphery of metagalaxy torsions. This sequence may represent a very long, probably infinite, row.

Therefore, the Universe is a system of interrelated plane cosmic vortexes. Initial orbital motion of any celestial object or their systems was caused by the ether orbital motion in a certain cosmic torsion, taking into account a possible tearing off of a local torsion from a periphery orbital massive of the ether and sharp braking of this torsion at the first moments of its existence due to the mass increase of this torsion. If the new-acquired orbital motion of the torsion produced the centrifugal force, which balanced the vortex gravitation force acting onto the local torsion, then this torsion should turn into a satellite of the parent torsion. Farther, the orbital motion of this cosmic object changed its characteristics in accordance with the laws presented in Section 3.2.

If the orbital speed of the satellite gets lower values, then the forces of the vortex gravitation should exceed the centrifugal forces, and this local torsion rushes toward the parent torsion center and finishes there its existence. If the orbital motion of the local torsion get a momentum with a high speed, then the centrifugal forces acting onto this torsion exceeds the gravitation forces, and this torsion must tear off from the parent torsion gravitation field and disappear in the cosmic space as a meteorite, asteroid, comet, etc. A newly-formed celestial object must permanently decrease the rotation velocity proportionally to the mass increase on the basis of the rotation angular momentum conservation law.The proposed principle of formation of torsionsatellites in the joint of two parent torsions allows explanation of the appearance of small planets - asteroids, comets; revolving of Venus in the direction opposite to that for other planets; 
revolving of some satellites in the direction opposite to that for the planets; considerable difference in the rotation speeds of Venus and its atmosphere; and other astronomy facts.

\subsection{BEGINNINGS OF SUBSTANCE}

In modern astrophysics, the origin of elementary particles is explained by thermonuclear reactions occurring in the star cores. That is, in the concluding stage of evolution, the star becomes unstable. Weakening nuclear reactions can not support inside the star such values of temperature and pressure which would ensure the stability of the enormous star mass. As the result, the gravitation, losing the control, causes the immediate compression (collapse) of the star. A giant ejection of energy as neutrino and impact waves, originating from the star interior, really blows away the outer star layers to the environment space, scattering heavy elements all over the galaxy. Such an ejection is usually called as nova outburst. Each nova outburst enriches the galaxy by negligibly small (trace) quantities of elements necessary for formation of the planets such as Earth, and further, for the origin and evolution of life in all the forms populating the Earth. It is obvious that the principle of beginnings of substance described above has an unconvincing character, because it treats the appearance of substance (in cosmic space) from substance (star cores). From the viewpoint of vortex gravitation and cosmogony, the possibility appears to explain the origin of any material particle using other properties of matter presented in this article. That is, in the peripheries of cosmic torsions, there exists a possibility of the origin of torsions of any volume, not only those of large celestial objects. Particularly, these could be the torsions of a size of nucleons, electrons, atoms, and other elementary particles. The number of these micro-torsions in the Universe can be infinite. Created in unlimited quantities, elementary particles in these micro-torsions serve as "bricks" of the tangible matter. In turn, the ether particles - amers - can be a "material" for creation of the elementary particles.

\subsection{RELATION BETWEEN ROTATION SPEED AND MASS}

On the basis of the principle of vortex cosmogony (Section 3.8), it is obvious that the rotation of a celestial object around its axis was resulted from the rotation of a corresponding ether torsion. Therefore, the higher is the cosmic torsion rotation speed, the higher is the speed of the celestial object created in the center of this torsion. On the other hand, it is evident from formula $\mathbf{1 0}$ that the torsion rotation speed determines the gravitation force in this torsion. The higher is the gravitation force, the higher is the degree of "sucking in" of the cosmic dust by this torsion and the greater should be the mass of the object created in the torsion center.

Consequently, comparing these qualities, one can see that the mass of a celestial object should be directly proportional to the speed of rotation of this object around its axis. Besides, the number of local torsions, in which satellites are created, also depends on the value of the parent torsion gravitation force. The above-indicated analytical interrelations between the planet masses and the number of satellites are confirmed by all the astronomy catalogues. It is known that the biggest and the "fastest" planet in the solar system is Jupiter. Saturn yields only to Jupiter in its rotation speed and mass. The order of magnitude of the masses of other planets also corresponds to the order of magnitude of the speeds of rotation around theirs axes.

It should be noted that Venus has a low rotation speed and a mass commensurate with that of Earth. This discrepancy with the above-said is explained by the fact that the planet masses are proportional not only to the ether pressure gradient and torsion rotation speed, but also they are proportional to the time of existence of this planet (torsion). That is, it is quite probable that Venus has weak gravitation force (which is confirmed by the absence of satellites), but it has a rather long term of existence, during which its rotation speed reduced in accordance with the angular momentum conservation law; this term was sufficient to accumulate the present volume. 
This suggestion proves additionally the fact that the Venus orbit plane coincides with the sun torsion central plane (Section 3.3). Consequently, the Venus torsion was formed on the outer, the very distant, periphery of the sun torsion, and not on a lateral one; further it moved by a spiral through all the sun torsion. Hence, Venus is the very old planet. The insignificant speed of rotation of the Sun is also explained by the term of existence of this star which essentially exceeds the term of existence of the planets (see Section 3.7). Therefore, the galaxies rotate around their axes with a speed which is less than that of the star rotation by several orders of magnitude.

\subsection{MASSES OF CELESTIAL OBJECTS}

Modern methods of calculation of masses of the planets and Sun are based on the Newton law, implying that the substance mass of a celestial body is directly proportional to the gravitation force directed to this body. Knowing the gravitation force and the body volume, researchers deduce the mass of the object from these data.According to the actual reference catalogues, some celestial objects have the following densities, which are rather doubtful:

Sun -1.4 ton/cub. $\mathrm{m}$.

Earth - $5.5-/$ -

Saturn - 0.7 - / -

As was shown above, the model of vortex gravitation excludes a direct dependence of the gravitation force on the object mass. Therefore, the densities and masses used today by astrophysicists have incorrect values, underestimated by an order of magnitude.

On the basis of the model of vortex gravitation, the possibility appears to determine the planet masses using other physical laws, particularly, the angular momentum conservation law. That is, comparing the rotation speed of ether with that of the celestial object (in one torsion), one can determine the mass increase as an inversely proportional dependence of the rotation speed reduction for the celestial object in the torsion center in comparison with the ether rotation speed. Such a calculation scheme is valid provided that the ether rotation braking is small and one may neglect this value. On the basis of this principle, the following densities are obtained:

Sun -30 ton/cub. $m$.

Earth - 23 ton/cub. m.

Mass of Earth $-2.5 \times 10^{25} \mathbf{k g}$.

\subsection{AGE OF CELESTIAL OBJECTS}

Modern theories of interior structure of celestial bodies, as well as planetary cosmogony, as an initial experimental basis for the estimation of the age of celestial objects, use the results of investigation of the age of mining rocks, sun neutrino, and other data obtained at studying the outer layer of the celestial object. Since, on the basis of the model of vortex cosmogony, the celestial objects were created by means of accumulation of cosmic matter, one can arrive at a conclusion that any inner layer should have its own age exceeding the age of the outer layer of this planet or star. Therefore, it is impossible to estimate the age of the interior substance or that of the celestial object on the whole from the data of study of outer rocks or any radiations from these rocks.

On the basis of vortex gravitation and creation of celestial objects, it is permissible to determine the age of planets by a mere division of the planet mass by a corresponding annual increase of the mass of this planet.

Taking into account the above-said, the following ages were obtained:

- Earth - 15.6 milliard years. 


\subsection{DARK MATTER}

It is known that in the middle last century, during the study of the galaxy structure, a discrepancy between the star distribution and the gravitation potential distribution has been found.Scientific opinion has divided into two groups. Some scientists state that the Newtonian theory of gravitation, developed on the basis of observation of planets in solar system, is not valid in larger astronomical scales. Most researchers agree that part of matter (30\%) does not emit photons, and therefore it is invisible. However, just this matter balances the gravitation potential in the galaxy. This invisible matter was called dark matter.

Obviously, in the theory of vortex gravitation, there are no difficulties in the explanation of this astronomy "paradox", because the world gravitation force does not depend on the masses of stars, but it only depends on the vortex rotation speed and the galaxy ether pressure gradient. The value of vortex gravitation in any point can be determined as described in Section 2. The obtained value of vortex gravitation completely balances the centrifugal forces of stars, and therefore there is no need to use that hypothetical dark matter. The above-discussed discrepancy of the masses of celestial objects with the gravitation, not only in the galaxies, but in the solar system too (see Section 4), additionally demonstrates the invalidity of the Newton's theory.

\subsection{EARTH EVOLUTION}

On the basis of vortex cosmogony, it is possible to reconstruct the physical state of our planet in the past. Using the formulas from Section 3.2, one determines that 1 milliard year ago, the planet of Earth had the following properties as compared to the present:

- the mass was $6 \%$ less,

- the radius of Earth was $2 \%$ less

- the day duration - 22.5 hours

- the vortex gravitation force was $4 \%$ higher, centrifugal forces were $13 \%$ higher, and therefore all the bodies were $9 \%$ lighter.

It should be noted that these properties directly affected on minerals, atmosphere, flora and fauna of that time. This is of high importance for researchers in various fields of natural history. Particularly, a weaker gravity mainly accounts for the existence of giant plants and animals that time.

The rotation speed of Earth round its axis must decrease, and hence a day must lengthen by $\mathbf{0 . 5 5}$ $x 0^{-5}$ sec a year.

Galileo was the first who had observed in 1695 the Earth rotation slowing down. From his observations, this slowing down was $2 \times 10^{-5}$ sec a year. Sixty years later, Kant has explained this effect by a flow friction. On the basis of the above-presented calculations, the Earth rotation slowing differs insignificantly from the results of astronomy observations of Galileo. The divergence in the results is accounted for by an error in the Earth mass determination [1] and its annual increase, as well as by the accuracy of astronomy observations. It is to be noted that, on the basis of vortex cosmogony, the Earth rotation slowing down has an explanation which differs absolutely from the hypothesis of Kant.

\section{CONCLUSION}

It is obvious that the above-presented principles of vortex gravitation and cosmogony are based on commonly-accepted basic physical laws. On the other hand, the classical law of universal gravitation is based on merely the evident equality of centrifugal forces $\mathbf{F c}$ and gravitation forces Fg. Further calculations to support this are connected with simple mathematical transformations: - the proportionality $\mathbf{V} \sim \mathbf{R}^{-2}$ is substituted into the equation $\mathbf{F g}=\mathbf{F} \mathbf{c}=\mathbf{m v} \mathbf{v}^{2} / \mathbf{R}$ from the $3^{\text {rd }}$ Kepler's law whence it is obtained that $\mathbf{F g} \sim \mathbf{m} / \mathbf{R}^{\mathbf{2}}$ 
In order to turn this ratio into a formula, I. Newton had advanced a hypothesis stating that all the bodies possessed the gravity with a force proportional to their masses. These masses were "prescribed" by a method of selection, and the gravitation constant was "introduced" in order that these masses were correct; it turned out to be necessary to give an absurd unity to this constant $-\mathbf{N} \mathbf{~ m}^{2} / \mathbf{k g}^{2}$

It is known that Newton himself was not sure in gravitation properties of physical bodies, and later he has suggested that the cosmic substance (ether) density change might be the cause of gravitation. However, no argumentation of the decrease of this density was presented so far. Huygens was known to call the Newton's hypothesis of gravitation properties of the bodies to be ridiculous.

In the theory of relativity, A. Einstein has grounded his more precise calculations on determination of the gravitation forces on the basic principles of I. Newton, i.e. on the centralsymmetrical action of the gravity forces and on the gravitation properties of celestial objects.

According to the Newton equation, the gravitation forces from any celestial object stretch over infinite distances, and, since the number of these objects is also infinite, classical theories of gravitation encounter a paradox called the Seliger paradox. That is, under these conditions, all the world space would be filled by infinite gravitation forces. The model of vortex gravitation removes this paradox, because the action of gravitation is limited by the size of the torsion.

Also, on the basis of the above-discussed classical theories, it is impossible to understand the cause of creation and motion of celestial objects. It is known that there exists the discordance in modern science concerning the speed of gravitation. From the classical physics, the speed of gravitation is infinite. According to the relativistic ideas, the speed of gravitation is equal to the speed of light. On the basis of vortex cosmology, the gravitation is obviously an inalienable property of any torsion. Therefore, the speed of gravitation corresponds to the speed of the appearance of a cosmic torsion.

It should be noted that many researchers advanced, and still continue to advance, the hypothesis on gravitation properties of the ether. However, up to date, nobody yet proposed a physical and mathematical ground of the interconnection of the ether motion with the change of its density, and the method for its calculation as well.

\section{ACKNOWLEDGEMENT}

The author thanks Dr. A. A. Velichko for his help with mathematical calculations in Section 2.

\section{REFERENCES}

1. Atsurovskiy, V. A. 1990. General ether-dynamics. Energoatomizdat. Moscow, Russia. Page 278.

2. Kiknadze, L. V. Yu. G. Mamaladze, 1979. Classical hydrodynamics for physicistsexperimentalists. Tbilisi University Press. Tbilisi, Georgia. Page 136.

3. Babichev, A. P., N. A. Babushkina 1991. Physical constants Handbook. Energoatomizdat. Moscow, Russia. Page 1232.

4. Kadyrov C. K, 2001. Physical Theory of Unified Field. Kyrgyz zer. Bishkek, Kyrgyzstan. Page 33.

5. Gulyaev, A. P. 1993 Astronomy calendar. Cosmosinform. Moscow, Russia. Page 285. 